\title{
Collinear resummations for the non-linear evolution in QCD at high energy
}

\author{
B. Duclouéa, , E. Iancu ${ }^{\mathrm{a}}$, A.H. Mueller ${ }^{\mathrm{c}}$, G. Soyez ${ }^{\mathrm{a}}$, D.N. Triantafyllopoulos ${ }^{\mathrm{d}}$ \\ ${ }^{a}$ Université Paris-Saclay, CNRS, CEA, Institut de physique théorique, 91191, Gif-sur-Yvette, France \\ ${ }^{b}$ Higgs Centre for Theoretical Physics, University of Edinburgh, Peter Guthrie Tait Road, Edinburgh EH9 3FD, UK \\ ${ }^{c}$ Department of Physics, Columbia University, New York, NY 10027, USA \\ ${ }^{d}$ European Centre for Theoretical Studies in Nuclear Physics and Related Areas (ECT*) \\ and Fondazione Bruno Kessler, Strada delle Tabarelle 286, I-38123 Villazzano (TN), Italy
}

\begin{abstract}
When computed to next-to-leading order in perturbative QCD, the non-linear Balitsky-Kovchegov (BK) equation for the high-energy evolution of the dipole-hadron scattering appears to be unstable. We show that this instability can be avoided by using the rapidity of the dense hadronic target (instead of that of the dilute dipole projectile) as the evolution time. Using this variable, we construct a collinearly-improved version of the BK equation, where the dominant radiative corrections to the kernel — those enhanced by double collinear logarithms — are resummed to all orders.
\end{abstract}

Introduction: High-energy evolution and gluon saturation. We would like to study the high-energy evolution of a hadron (proton or nucleus) wavefunction in the vicinity of saturation, i.e. in the high parton density regime where gluon occupation numbers are of order $1 / \alpha_{s}$ and non-linear effects like gluon recombination play an important role. This evolution proceeds via the successive emission of soft gluons with smaller and smaller longitudinal momentum fraction $x=k^{-} / P^{-}$; here, $k^{-}$is the (light-cone) longitudinal momentum of the emitted gluon and $P^{-}$is the respective momentum of the parent nucleon, assumed to be an energetic left mover. The phase-space for this evolution is controlled by the rapidity difference $\eta \equiv \ln \left(P^{-} / k^{-}\right)=\ln (1 / x)$. The probability for one soft gluon emission is of order $\bar{\alpha}_{s} \eta$, with $\bar{\alpha}_{s} \equiv \alpha_{s} N_{c} / \pi$. When $\bar{\alpha}_{s} \eta \gtrsim 1$, gluon emissions must be iterated, which one can do by solving a evolution equation in $\eta$, that we intend to construct. With decreasing $x$, or increasing $\eta$, the gluon density grows, hence saturation occurs for smaller and smaller gluon transverse sizes $\Delta x_{\perp}$, or, equivalently, for larger and larger transverse momenta $k_{\perp} \sim 1 / \Delta x_{\perp}$. The typical transverse momentum for which a gluon with energy fraction $x$ reaches saturation is known as the saturation momentum $Q_{s}(\eta)$. The previous discussion shows that this scale is increasing with $\eta$. For sufficiently large $\eta$ at least, this increase is exponential: $Q_{s}^{2}(\eta) \simeq Q_{0}^{2} \mathrm{e}^{\lambda_{s} \eta}$, where the saturation exponent $\lambda_{s}$ is a number of $O\left(\bar{\alpha}_{s}\right)$, to be obtained from solutions to the aforementioned evolution equation.

Email addresses: bertrand.ducloue@ed.ac.uk (B. Ducloué), edmond.iancu@ipht.fr (E. Iancu), ahm4@columbia.edu (A.H. Mueller), gregory.soyez@ipht.fr (G. Soyez), trianta@ectstar.eu (D.N. Triantafyllopoulos) 
Deep inelastic scattering at small Bjorken $x$. To study this evolution in a gauge-invariant manner, we consider the scattering between the hadron and a small projectile, with transverse resolution of the order of the saturation scale. Two important examples are deep inelastic scattering (DIS) at small Bjorken $x_{\mathrm{Bj}}$ and particle production in proton-nucleus $(p A)$ collisions. For definiteness, here we shall mostly refer to DIS. The Bjorken $x_{\mathrm{Bj}}$ variable is defined as $x_{\mathrm{Bj}} \equiv \frac{Q^{2}}{2 P \cdot q}=\frac{Q^{2}}{2 P^{-} q^{+}}$, where the second equality holds in a frame where the virtual photon $\gamma^{*}$ is an energetic right-mover, with 4-momentum $q^{\mu} \equiv\left(q^{+}, q^{-}, \boldsymbol{q}_{\perp}\right)=\left(q^{+},-Q^{2} / 2 q^{+}, \mathbf{0}_{\perp}\right)$, whereas the proton is a left-mover with $P^{\mu}=\delta^{\mu-} P^{-}$. At high-energy or small Bjorken $x_{\mathrm{Bj}}$, this process admits a dipole factorization: $\gamma^{*}$ fluctuates into a quark-antiquark pair in a colour-singlet state (the "dipole"), which then inelastically scatters off the proton target. Indeed the condition $x_{\mathrm{Bj}} \ll 1$ implies that the lifetime $\Delta x^{+} \simeq 2 q^{+} / Q^{2}$ of the $q \bar{q}$ fluctuation is much larger than the longitudinal extent $1 / P^{-}$of the target.

The dipole-proton interaction is controlled by the target gluons with longitudinal momenta $k^{-} \gtrsim Q^{2} / 2 q^{+}$, which fully overlap in time with the $q \bar{q}$ fluctuation $\left(1 / k^{-} \lesssim \Delta x^{+}\right)$. The fraction $x=k^{-} / P^{-}$of the softest such gluons coincides with $x_{\mathrm{Bj}}$, so the latter fixes the rapidity phase-space $\eta=\ln (1 / x)$ for the target evolution. From now on we shall identify these two variables, $x_{\mathrm{Bj}} \equiv x$, and use the simpler notation $x$ for both.

Target vs. dipole evolution. Thus, the physical problem that we are interested in - the evolution of the proton wavefunction with decreasing $x-$ is tantamount to computing the $x$-dependence of the DIS structure functions for fixed $Q^{2}$ (eventually chosen of the order of $Q_{s}^{2}(\eta)$, to study gluon saturation in the target). However, this problem is complicated, due to the need to compute gluon emissions in a dense environment, and so far it has been worked out only to leading order (LO) accuracy, leading to the JIMWLK equation [1].

A simpler approach is to follow the evolution of the dipole. Soft gluon emissions from the dipole occur like in the vacuum and non-linear effects exclusively refer to multiple scattering. This viewpoint leads to the Balitsky hierarchy (in particular, to the BK equation), which is currently known to next-to-leading-order (NLO) accuracy $[2,3,4,5]$. Our purpose is to go beyond LO, so we shall adopt the dipole evolution in what follows. But this comes with a drawback: the NLO version of the BK equation turns out to be unstable [6].

This instability can be traced back to two facts: (i) The rapidity difference $Y \equiv \ln \left(q^{+} / q_{0}^{+}\right)$between the projectile and the softest gluons in the dipole wavefunction is different from the rapidity $\eta=\ln (1 / x)$ of the target. (ii) The LO evolution in $Y$ can violate the correct time ordering of the successive gluon emissions.

Concerning point ( $i$ ), we observe that the softest gluons from the dipole wavefunction which matter for the scattering are those with a transverse momentum $\sim Q_{0}$ and a lifetime $2 q_{0}^{+} / Q_{0}^{2} \sim 1 / P^{-}$; this implies

$$
Y=\ln \frac{q^{+}}{q_{0}^{+}}=\ln \frac{2 q^{+} P^{-}}{Q_{0}^{2}}=\ln \frac{1}{x}+\ln \frac{Q^{2}}{Q_{0}^{2}} \equiv \eta+\rho .
$$

The difference $Y-\eta=\rho \equiv \ln \left(Q^{2} / Q_{0}^{2}\right)$ is quite large for the interesting values $Q^{2} \sim Q_{s}^{2}(\eta)$ : the relative difference $(Y-\eta) / \eta \sim \lambda_{s}$ is of $O\left(\bar{\alpha}_{s}\right)$, so it formally matters at NLO and beyond.

Concerning point $(i i)$, we note that, in the LO evolution with $Y$, the successive gluon emissions are explicitly ordered in longitudinal momenta, $q^{+} \gg k_{1}^{+} \gg k_{2}^{+} \gg \ldots \gg q_{0}^{+}$, and implicitly ordered in lifetimes

$$
\frac{2 q^{+}}{Q^{2}} \gg \frac{2 k_{1}^{+}}{k_{1 \perp}^{2}} \gg \frac{2 k_{2}^{+}}{k_{2 \perp}^{2}} \gg \ldots \gg \frac{2 q_{0}^{+}}{Q_{0}^{2}},
$$

since daughter gluons must live shorter than their parents. Although implicitly assumed, time-ordering is not explicitly enforced in the LO BK equation. In fact, it is violated by the typical emissions, whose transverse momenta obey $Q^{2} \gg k_{1 \perp}^{2} \gg k_{2 \perp}^{2} \gg \ldots \gg Q_{0}^{2}$ and which give the dominant, double-logarithmic, contributions to the evolution of the dipole amplitude: a series of powers of $\bar{\alpha}_{s} Y \rho$. Such violations are unphysical and are corrected by higher-order perturbative corrections to the evolution in $Y$. Yet, the associated corrections are parametrically large and spoil the convergence of the weak coupling expansion.

To understand that, notice that the effect of the time-ordering condition (2) is to reduce the rapidity phase-space available for the evolution from $Y$ to $Y-\rho \equiv \eta$. Accordingly, the correct double-logarithmic approximation should be a series in powers of $\bar{\alpha}_{s}(Y-\rho) \rho$, instead of the LO series in powers of $\bar{\alpha}_{s} Y \rho$. The difference between the two corresponds to a tower of series of radiative corrections enhanced by the double 
"anti-collinear" $\operatorname{logarithm} \rho^{2}$ : the dominant series includes all powers of $\bar{\alpha}_{s} \rho^{2}$, the subdominant one, those of $\bar{\alpha}_{s}^{2} \rho^{2}$, etc. In particular, the NLO BK equation includes the first (negative) contribution proportional to $\bar{\alpha}_{s} \rho^{2}[2]$ which makes the evolution unstable [6] and hence unsuitable for physical studies.

Collinear resummations in $Y$. The dominant series in powers of $\bar{\alpha}_{s} \rho^{2}$ can be resummed to all orders by enforcing time-ordering in the LO BK evolution [7, 8]. Two "collinearly improved" BK equations have been proposed [7, 8], with seemingly promising results: the ensuing equations are stable [8,9], they can be extended to full NLO accuracy [9] and they allow for good fits to the HERA data for DIS at small $x[10,11]$.

A more recent study has revealed that these apparent successes are deceiving [12]. The numerical studies in $[9,10,11]$ have been presented in terms of $Y$ instead of the physical rapidity $\eta=Y-\rho=\ln (1 / x)$. Also, in the DIS fits in $[10,11]$, the variable $Y$ has been abusively interpreted as $\ln (1 / x)$. When translating the respective results from $Y$ to $\eta$, one finds [12] an unacceptably large resummation-scheme dependence, that should be attributed to the uncontrolled, subleading, double-logarithmic corrections. This large scheme dependence forbids any physical interpretation of the results. The problem is further complicated by the fact that the resummed BK evolution in $Y$ cannot be formulated as an initial-value problem [12].

Recasting dipole evolution in terms of $\eta$. These difficulties with the evolution in $Y$ can be avoided by using $\eta$ as an "evolution time" for the dipole projectile [12]. This may not look very natural (recall that $\eta$ is the rapidity of the target), yet it can be unambiguously implemented in perturbation theory, via a change of variables $Y \rightarrow \eta \equiv Y-\rho$. And indeed, Ref. [12] has used this trick to deduce the NLO BK equation in $\eta$ from the respective equation in $Y$ [2]. When the gluon emissions are ordered in $\eta$, they automatically obey the correct time-ordering: indeed, $\eta=\ln \left(P^{-} / k^{-}\right)$, hence ordering in $\eta$ is tantamount to ordering with increasing $k^{-}$, or decreasing $\Delta x^{+}=1 / k^{-}$. Besides, $\eta=\ln (1 / x)$ is the right variable to be used in phenomenological studies of DIS. Last but not least, the evolution in $\eta$ is naturally formulated as an initial value problem.

Since the proper time-ordering is now automatic, the evolution in $\eta$ is not affected by the large anticollinear logarithms present in the evolution in $Y$. On the other hand, the ordering in $k^{+}$is not enforced anymore and it can be violated by collinear emissions, where the daughter gluon has a much larger transverse momentum than the parent one. Such collinear emissions are atypical in the DIS context (they don't matter for the double-logarithmic approximation), hence they are less problematic. E.g., they yield doublelogarithmic corrections to the BK kernel (starting with NLO), but not to the solution to the BK equation [12]. Besides, they are suppressed by saturation, which freezes the evolution for emissions with $k_{\perp} \lesssim Q_{s}(\eta)$. This being said, the effects of the double collinear logarithms in the kernel are still quite large and moreover they accumulate during the evolution. This eventually translates into an instability at large enough $\eta$ [12].

Collinear resummations in $\eta$. As for the evolution in $Y$ [7], one can avoid this instability via an all-order resummation leading to an evolution non-local in $\eta$ [12]. Unlike what happens with the resummations in $Y$, the scheme dependence for the resummations in $\eta$ is small and in agreement with the expected perturbative accuracy of the resummed equation. Specifically, the collinearly-improved BK equation in $\eta$ reads [12]

$$
\frac{\partial S_{x y}(\eta)}{\partial \eta}=\int \mathrm{d}^{2} z \frac{\bar{\alpha}_{s}\left(r_{\min }\right)}{2 \pi} \frac{(\boldsymbol{x}-\boldsymbol{y})^{2}}{(\boldsymbol{x}-\boldsymbol{z})^{2}(z-\boldsymbol{y})^{2}}\left[S_{x z}\left(\eta-\delta_{x z ; r}\right) S_{z y}\left(\eta-\delta_{z y ; r}\right)-S_{x y}(\eta)\right],
$$

Here, $S_{x y}$ is the elastic $S$-matrix for a dipole with transverse coordinates $\boldsymbol{x}$ and $\boldsymbol{y}$, for the quark and the antiquark respectively. The emission of a soft gluon with transverse position $z$ is viewed as the splitting of the original dipole $(\boldsymbol{x}, \boldsymbol{y})$ into two daughter dipoles $(\boldsymbol{x}, \boldsymbol{z})$ and $(z, \boldsymbol{y})$. Besides using the target rapidity $\eta$ (instead of the dipole rapidity $Y$ ), Eq. (3) differs from the LO BK equation via the rapidity shift, defined as

$$
\delta_{x z ; r} \equiv \max \left\{0, \ln \frac{r^{2}}{|\boldsymbol{x}-\boldsymbol{z}|^{2}}\right\}
$$

with $r \equiv|\boldsymbol{x}-\boldsymbol{y}|$ and similarly for $\delta_{z y ; r}$. This is non-zero only for emissions in which one of the daughter dipoles is smaller than the parent one. By Taylor-expanding the rapidity shifts and iteratively using the BK equation for $\partial S / \partial \eta$, one generates the whole series of (leading) double collinear logarithms. The running 

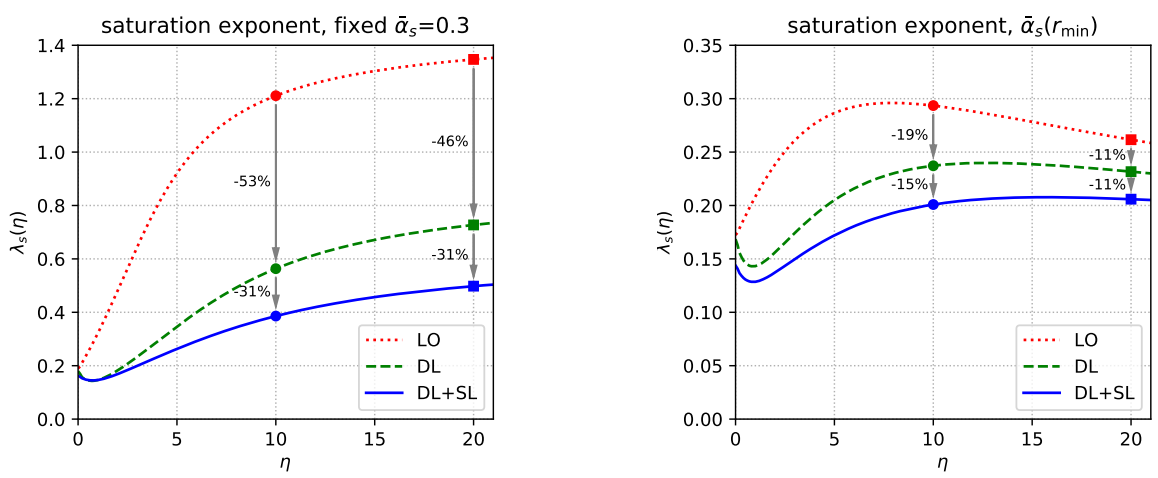

Fig. 1: The saturation exponent $\lambda_{s}(\eta)$ predicted by Eq. (3) with Gaussian initial condition $S_{0}(r)=\exp \left[-r^{2} Q_{0}^{2}\right]$ with $Q_{0}^{2}=1 \mathrm{GeV}^{2}$ and with both fixed and running coupling. The lines denoted as DL (green, dashed) and LO (red, dotted) are obtained with and without the rapidity shift, respectively. The DL+SL (continuous, blue) line corresponds to additionally resumming DGLAP-like single logs $[8,12]$.

coupling corrections are known to be important and can be minimised by using a one-loop running coupling $\bar{\alpha}_{s}\left(r_{\text {min }}\right)$ with the scale set by the size $r_{\min }$ of the smallest dipole: $r_{\min } \equiv \min \{|\boldsymbol{x}-\boldsymbol{y}|,|\boldsymbol{x}-\boldsymbol{z}|,|\boldsymbol{z}-\boldsymbol{y}|\}$. Eq. (3) has to be solved as an initial value problem, with the initial condition $S_{x y}\left(\eta \leq \eta_{0}\right)=S_{x y}^{(0)}$. It is possible to extend this equation to full NLO accuracy by adding the missing pure $\bar{\alpha}_{s}^{2}$ corrections [12].

In Fig. 1 we show the results of Eq. (3) for the exponent $\lambda_{s}(\eta) \equiv \mathrm{d} \ln Q_{s}^{2} / \mathrm{d} \eta$, with $Q_{s}(\eta)$ obtained from the condition that $S(\eta, r)=0.5$ when $r=2 / Q_{s}(\eta)$. The effect of the resummation is important, especially for a fixed coupling. When the coupling runs, the whole evolution slowes down considerably, but the seemingly modest, additional, reduction $(\sim 20 \%)$ in $\lambda_{s}$ due to the resummation is essential to optimally accommodate the small- $x$ HERA data. This is demonstrated in [13], where very good fits for the inclusive DIS data at $x \leq 0.01$ were obtained by using the collinearly improved BK equation in $\eta$ (with a further inclusion of DGLAP logs, cf. Fig. 1), together with a physically motivated model for the initial condition at $x_{0}=0.01$.

Acknowledgements. Part of the work of B.D, E.I. and G.S. has been supported by the Agence Nationale de la Recherche project ANR-16-CE31-0019-01. Part of the work of B.D has been supported by the ERC Starting Grant 715049 “QCDforfuture”.

\section{References}

[1] F. Gelis, E. Iancu, J. Jalilian-Marian, R. Venugopalan, The Color Glass Condensate, Ann.Rev.Nucl.Part.Sci. 60 (2010) 463-489, arXiv:1002.0333.

[2] I. Balitsky, G. A. Chirilli, Next-to-leading order evolution of color dipoles, Phys.Rev. D77 (2008) 014019, arXiv:0710.4330.

[3] I. Balitsky, G. A. Chirilli, Rapidity evolution of Wilson lines at NLO, Phys.Rev. D88 (2013) 111501, arXiv: 1309.7644.

[4] A. Kovner, M. Lublinsky, Y. Mulian, NLO JIMWLK evolution unabridged, JHEP 08 (2014) 114, arXiv: 1405.0418.

[5] M. Lublinsky, Y. Mulian, High Energy QCD at NLO: from light-cone wave function to JIMWLK evolution, JHEP 05 (2017) 097, arXiv: 1610.03453.

[6] T. Lappi, H. Mäntysaari, Direct numerical solution of the coordinate space Balitsky-Kovchegov equation at next to leading order, Phys.Rev. D91 (7) (2015) 074016, arXiv: 1502.02400

[7] G. Beuf, Improving the kinematics for low-x QCD evolution equations in coordinate space, Phys.Rev. D89 (2014) 074039, arXiv: 1401.0313.

[8] E. Iancu, J. Madrigal, A. Mueller, G. Soyez, D. Triantafyllopoulos, Resumming double logarithms in the QCD evolution of color dipoles, Phys.Lett. B744 (2015) 293-302, arXiv : 1502.05642.

[9] T. Lappi, H. Mäntysaari, Next-to-leading order Balitsky-Kovchegov equation with resummation, Phys. Rev. D93 (9) (2016) 094004, arXiv: 1601.06598.

[10] E. Iancu, J. D. Madrigal, A. H. Mueller, G. Soyez, D. N. Triantafyllopoulos, Collinearly-improved BK evolution meets the HERA data, Phys. Lett. B750 (2015) 643-652, arXiv: 1507.03651.

[11] J. L. Albacete, Resummation of double collinear logs in BK evolution versus HERA data, Nucl. Phys. A957 (2017) 71-84, arXiv: 1507.07120.

[12] B. Ducloué, E. Iancu, A. H. Mueller, G. Soyez, D. N. Triantafyllopoulos, Non-linear evolution in QCD at high-energy beyond leading order, JHEP 04 (2019) 081, arXiv: 1902.06637.

[13] B. Ducloué, E. Iancu, G. Soyez, D. N. Triantafyllopoulos, HERA data and collinearly-improved BK dynamics, arXiv: 1912 . 09196. 